\title{
Renaissance et Humanisme en Slovaquie
}

EVA FRIMMOVÁ

Résumé: Cet article constitue un survol analytique de l'impact des idées nouvelles sur la culture et le monde intellectuel en Slovaquie durant le période renaissante. Les contacts sont nombreux entre les humanistes, les intellectuels et les scientifiques autant à Presbourg que dans les villes secondaires. La conjonction de l'humanisme et de la réforme sert de point de départ au développement d'une conscience nationale slovaque.

$\mathrm{A}^{\mathrm{n} \text { raison de la variété des conditions économiques, sociales, politiques et }}$ Eculturelles, le bouillonnement d'idées, de culture et de société, formant le passage entre le Moyen-Age et les temps modernes, est unique dans chaque pays européen. Dans toute étude de cette époque, il est important, pour éviter des interprétations parfois trop simplistes, de comprendre la complexité des influences de l'humanisme et de la Renaissance sur les cultures nationales et, en même temps, d'en discerner les particularités.

Le territoire de la Slovaquie, intégré dès le commencement du onzième siècle à la Hongrie multinationale, ${ }^{1}$ était à la fin du Moyen-Age et au début des temps modernes un pays relativement développé. Du point de vue économique, par exemple, il garantissait, grâce à la richesse de ses mines d'or, d'argent et de cuivre, le quart du budget du royaume hongrois. ${ }^{2}$ Son importance stratégique s'est multipliée après la défaite tragique près de Moháč en 1526, où les Turcs ont vaincu les Hongrois (et le jeune roi Louis II Jagellon est mort). Après cette défaite, une expansion osmane incessante a commencé sur une grande partie de la Slovaquie. ${ }^{3}$ L'administration centrale, y compris la chambre royale, s'est déplacée de Buda à Presbourg, ${ }^{4}$ qui 
est alors devenue le lieu du couronnement des rois hongrois. ${ }^{5}$ En même temps, on a relocalisé à Trnava ${ }^{6}$ le secrétariat de l'archevêché d'Esztergom qui couvrait presque tout le territoire de la Slovaquie du point de vue de l'administration de l'Église.

Les villes libres royales et les villes minières avec une forte représentation d'origine allemande ont exercé une influence importante sur le développement général du pays. ${ }^{8} \mathrm{D}$ 'après les recherches démographiques, on suppose qu'au commencement du seizième siècle la Slovaquie comptait environ 550000 habitants, ${ }^{9}$ mais à la fin du même siècle ce nombre atteignait les 800000 à $900000 .{ }^{10}$ Sous le règne de Mathias Corvin Hunyadi (1458-1490), le territoire de la Slovaquie a joui d'une prospérité inhabituelle et remarquablement propice à la stabilisation du pays et à la créationč de conditions favorables à l'évolution des sciences, de la culture et des arts. La situation a changé après la défaite près de Moháč. ${ }^{11}$ Par la suite a commencé un drame de 20 ans sur le droit de succession entre Ferdinand $1^{\text {er }}$ de Habsbourg (1526-1564) ${ }^{12}$ et Ján Zápol'ský. ${ }^{13}$ Enfin, la Slovaquie est devenue une composante de la monarchie de la famille des Habsbourg. Les contradictions de l'époque se sont donc manifestées avec force, sans égard à la menace osmane, qui est restée très présente pendant plus que 150 ans. Le mécontentement, le désir du renversement du règne légal de l'empire ainsi que le postulat de la liberté de confession se sont reflétés dans les révoltes contre la maison des Habsbourg. ${ }^{14}$ Et c'est justement sur le territoire de la Slovaquie qu'une grande partie de ces événements a eu lieu.

Malgré cet arrière-plan politique et économique, qui n'était certes pas le plus favorable, et un certain retard relativement aux autres pays européens, on a pu assister en Slovaquie à une évolution de la culture et de l'érudition, comparable dans certaines régions à la situation générale de l'Europe centrale. Plusieurs facteurs y ont contribué, qui ont permis l'acceptation et la transformation des idées nouvelles sur le territoire slovaque et assuré l'influence importante de ces idées sur la culture locale. En ce qui concerne l'évolution du climat politique aux quinzième et seizième siècles, on peut remarquer deux nouvelles tendances selon la région: premièrement, une influence de la Renaissance italienne; et deuxièmement une pénétration de l'humanisme et de la Réforme allemande.

La première véritable étape de la Renaissance slovaque, aussi bien que dans le contexte religieux de la Devotio moderna, remonte au règne de Mathias Corvin et des Jagellons dans la deuxième moitié du quinzième siècle et persiste jusqu'au début du seizième siècle. Elle n'implique que les hautes 
couches de la société. La deuxième vague de l'humanisme tardif s'étend du seizième jusqu'au milieu du dix-septième siècle dans les classes moyennes, surtout dans le patriciat des villes libres royales. Ici, l'humanisme d'Érasme et de Melanchthon pénètre graduellement les mentalités, à la faveur de la Réforme, considérée par certains auteurs comme une branche de l'humanisme, appelé "Humanisme de Réforme." La Renaissance renouvelle la conception du monde et de l'homme; le déploiement harmonieux de la personnalité humaine nourrit les rêves des humanistes et fonde leurs doctrines. Humanisme et Réforme changent donc la vie sociale, surtout l'évolution de la culture, de la langue et de la littérature nationales.

En Slovaquie, la naissance d'une littérature formée par l'humanisme et les nouvelles idées religieuses remonte surtout aux années 1500-1540, sa plus grande floraison allant de 1540 à 1620. Au-delà de ces dates, son retentissement jusqu'en 1650 correspond plutôt à un maniérisme littéraire qui signale le début de la littérature baroque. ${ }^{15}$ Les recherches de Jozef Minárik et de Jozef Kuzmík ${ }^{16}$ renvoient aux 1400 lettrés qui, résidant à l'extérieur des frontières durant cette période, provenaient de la Slovaquie ou qui, venus d'ailleurs, avaient été invités à y travailler, à titre de recteurs des écoles, par exemple. Un grand nombre d'entre eux sont restés à l'étranger après leurs études. Pour déterminer les causes de ce phénomène, il faut, en première ligne, prendre en considération un certain nombre de facteurs sociaux et culturels comme la transformation de la situation des clercs et du prestige social des lettrés, bien qu'on ait senti l'absence du clergé et des intellectuels. Tous les groupes de lettrés provenant du territoire slovaque ou encore ceux-là qui entretenaient avec la Slovaquie des liens actifs concouraient à son développement culturel ou scientifique; c'est pourquoi, peu importe leur lieu de travail ou d'études, on les tient pour des auteurs reliés à l'histoire de la Slovaquie.

Dès le Moyen Age, il faut faire état d'influences italiennes sur le territoire hongrois; au quatorzième siècle, la cour du roi Charles Robert d'Anjou (1308-1342) ${ }^{17}$ en est imprégnée; au début du quinzième siècle, ces influences transitent surtout par la Croatie et la Transilvanie. ${ }^{18}$ Elles se sont intensifiées durant la deuxième moitié du quinzième siècle sous le règne de Mathias Corvin. Si l'on peut parler à cette époque-là de Renaissance et d'humanisme en Slovaquie, ces développements sont dûs avant tout à la fondation et à la brève existence de l'Université "Istropolitana" à Presbourg entre 1465-1490. ${ }^{19}$ Cet établissement avait été fondé d'après le modèle de l'Université de Bologne et avait pour but d'assurer la formation d'hommes 
érudits pour l'administration de l'État. En même temps, elle devait jouer un rôle dans la prévention contre la propagation des idées hérétiques "hussitescalixtines" qui pénétraient en Slovaquie par la Bohême à travers la Moravie. ${ }^{20}$ C'est Johannes Vitéz de Sredna, archevêque d'Esztergom, qui a profité le plus de la création de cette université: il y était chancellier. Le vice-chancellier était Juraj Schomberg, prévôt de Presbourg. L'organisation des quatre facultés a été conçue selon les idées humanistes et son enseignement selon une méthodologie considérée moderne à l'époque; on y emploie l'élément laïque, et on y fait la promotion d'une nouvelle attitude devant la vie et devant les doctrines de l'Église, ainsi que d'un intérêt pour les sciences naturelles. Des personnages d'importance y ont enseigné, comme le professeur d'astrologie Johann Müller Regiomontanus de Königsberg (1436-1476), qui s'occupait alors, comme son professeur Georg Peuerbach de Vienne, de l'édition critique de l'astronomie géocentrique Almageste, mégalé syntaxis de Claude Ptolémée et qui préparait le manuel astronomique Astronomiae Horologium, Tabulae primi mobilis, l'horoscope pour Mathias Corvin. Il a publié plusieurs calendriers astronomiques - Ephemerides. ${ }^{21} \mathrm{Il}$ a eu pour successeur à Presbourg son disciple Martin Bylica de Okulsz (1433-1493). Il y a beaucoup d'indices qui confirment l'existence de facultés de médecine et de droit. On note les nom de nombreux professeurs, comme Peter de Verona, Doctor artium et medicinae, Erasmus Adlman, Martin Bylica, qui enseignait aussi la médecine, Johannes Gattus, Doctor decretorum et magister sacrae theologiae; à la faculté de théologie, on peut citer Matthias Gruber de Medling et Laurentius de Krompachy (en Slovaquie), sauf le dominicain Johannes Gattus, professeur à la fois de théologie et de droit.

A l'exception de la cour humaniste royale de Mathias Corvin, il faut mentionner en rapport avec la Slovaquie les activités de sa deuxième femme Béatrice d'Aragon, princesse de Naples (1457-1508), éduquée dans l'esprit de la Renaissance. ${ }^{22}$ Le couple royal séjournait souvent sur le territoire slovaque, surtout à Presbourg, Komárno et dans les villes minières au centre du pays. ${ }^{23}$ Par la suite, on doit noter l'acceptation d'éléments laïcs dans le cadre de la Devotion moderna, chez des personnages éminents comme le roi Mathias Corvin, l'archevêque Johannes Vitéz, son neveu Johannes Pannonius, le théologue sicilien Giovanni Gatti, Galeotto Marzio, évêque de Zagreb, et Johannes Turzo. Ces personnages survivent dans l'ouvrage célèbre de Galeotto Marzio De egregie sapienter et iocose dictis ac factis Mathiae regis. ${ }^{24}$ 
En esquissant le tableau complexe de cette époque-là, il est nécessaire de caractériser plusieurs de ces personnages de la vie politique, culturelle et ecclésiastique, de même que les activités des institutions à Presbourg ${ }^{25}$ et dans d'autres régions de la Slovaquie. Cet arrière-plan est intellectuellement fort riche, notamment dans le centre du pays, dans les villes de Kremnica, Banská Štiavnica, Banská Bystrica et, à l'Est de la Slovaquie, dans les villes de Levoča, Bardejov et Košice. Dès les temps anciens, un grand nombre d'étudiants de Slovaquie suivaient des cours dans les universités étrangères, en particulier en Italie, mais aussi dans les villes plus proches, comme Cracovie, ${ }^{26}$ Vienne ${ }^{27}$ et Prague. ${ }^{28}$ Outre les monuments littéraires, les notes et les protocoles de divers bureaux et lieux crédibles - "loca credibilia" ont pour nous une grande valeur; ils nous informent, encore aujourd'hui, sur le haut niveau relatif de l'expression écrite en Slovaquie. Ensuite, il faudrait analyser le matériel d'archives et les fonds de livres qui attestent l'existence des anciennes bibliothèques appartenant à des curés et des pasteurs, des baillis, des notaires, des scribes, des recteurs, des maîtres, des professeurs, des précepteurs et d'autres lettrés, de même que d'une variété d'institutions: monastères, écoles, confréries ou bibliothèques paroissiales et de la noblesse. ${ }^{29}$ Leur contenu thématique signalerait une forte influence des idées modernes et un grand intérêt pour l'antiquité et les sciences naturelles. ${ }^{30} \mathrm{En}$ même temps, une production originale apparaît; c'est par exemple le cas du scribe du couvent bénédictin à Štôla qui a achevé en 1410 l'oeuvre d'un moine inconnu, le Malogranatum, devenu le manuel principal du mouvement de la Devotio moderna. ${ }^{31}$

Ces repères indirects mettent en évidence qu'à cette époque-là le milieu aisé possède une culture dont le seizième siècle a profité considérablement. Dès le début, on trouve des personnages importants dans la vie non seulement culturelle, mais aussi politique et ecclésiastique, qui oeuvrent pendant de brèves ou de longues périodes en Slovaquie et ainsi influencent la formation de la culture locale. On peut mentionner parmi ceux-ci Girolamo Balbi, un Italien, dont certains poèmes, lettres et discours touchent, d'une manière ostensible, le territoire slovaque. Il a été surtout actif à la cour royale en tant que précepteur et plus tard délégué et orateur; en 1515, il devient même prévôt du chapitre de Presbourg. Dans son ouvrage intitulé De rebus Turcicis, il signale l'immense menace turque pour le monde chrétien. Dans le poème "Ad Johannem Baptistam Calvum sodalem," il décrit, avec ironie, la vie luxuriante et débauchée de la haute noblesse hongroise, ce qu'on lui reprochera plus tard. ${ }^{32}$ Conrad Sperfogel de Suisse, bailli à Levoča, a noté 
dans son Diarium les événements importants de la région de Spiš pendant les années 1516-1536; l'humaniste anglais Leonhard Coxe en tant que recteur à Levoča et Košice propageait des principes éducatifs modernes dans ses oeuvres intitulées Libellus de erudienda juventute (Cracovie 1526) et Methodus humaniorum studiorum (Cracovie 1523). ${ }^{33}$ Pour les autres, il faut citer l'archevêque d'Esztergom et le primat hongrois Mikuláš Oláh, originaire de la Transilvanie, ${ }^{34}$ qui a encouragé la recatholicisation du pays après le concile de Trente (1542-1564). De même, il s'est occupé de l'histoire hongroise comme une suite des auteurs Štefan Brodarič ou Štefan Taurinus etc... Valentin Eck de Lindau en Bavière, recteur à Košice et à Bardejov, a rédigé des manuels scolaires importants comme le De arte versificandi (Cracovie 1515) et le De ratione legendi auctores libellus (Cracovie 1523). ${ }^{35}$

La Renaissance pose l'homme au centre des pensées et de l'activité humaine, et c'est pourquoi elle trouve à se refléter dans tous les domaines de la vie sociale et culturelle. Grâce à elle, quelque chose d'extraordinaire et de nouveau entre dans l'histoire et établit une rupture avec le Moyen-Age. On n'a pas réussi à mesurer à l'échelle de l'Europe ces caractéristiques principales, surtout pour les pays transalpins, ni d'ailleurs l'ensemble des phénomènes d'idées et autres qui peuvent être inclus dans la notion de Réforme. ${ }^{36}$ Cependant, l'opposition à l'ordre médieval et à la rigidité unilatérale est moins perceptible sur le territoire de la Slovaquie. Il s'agit plutôt d'une progression lente se mêlant à de fortes traditions. L'intérêt pour les sciences touchant aux disciplines humaines, en général "humniora litteraria", est confiné selon les conseils d'Érasme au domaine de l'érudition cultivée, les "bonae litterae"; cet intérêt est donc propre au cercle des lettrés. $S$ 'appuyant pourtant sur un immense désir de savoir, il révise les connaissance anciennes, il critique les gloses et les commentaires médiévaux et il s'oriente vers une évolution parfaite de l'esprit humain. ${ }^{37}$ Les premiers humanistes n'étaient ni protestants ni païens, ils ne souhaitaient que l'élévation harmonieuse de l'homme, son érudition et sa vie ecclésiastique active. Tout cela aurait dû se manifester dans une amélioration tangible de la société.

C'est relativement tôt que se publient, en Slovaquie, des oeuvres politiques et philosophiques qui proposent une réflexion théorique sur la gestion convenable de l'État et sur son administration, sur les vertus du souverain et sur l'attitude de l'empereur à l'égard des lois. Le traité de Valentin Eck à Bardejov De reipublicae administratione, ${ }^{38}$ dédié à Alexius Turzo, qui était son mécène et le futur secrétaire de Louis II, paraît dès 1520. Cependant, par ses ouvrages Libellus de partibus reipublicae et causis mutationum 
regnorum imperiorumque (Vienne 1560; 348 distiques) et magistratu politico (Lipsiae 1574; 1316 distiques), ${ }^{39}$ Martin Rakovský occupe la plus importante place. Dans son poème philosophique et didactique, Libellus de partibus reipublicae, il présente une analyse philosophique de la société contemporaine et une réflexion sur son amélioration. Ce texte est fortement influencé par la Politique d'Aristote et par les commentaires de Melanchthon touchant le point de vue chrétien. ${ }^{40} \mathrm{Ce}$ poème est considéré en Hongrie comme une oeuvre unique et il est digne de la plus haute admiration, et pour sa forme et pour son contenu. Comme monarchiste, Rakovský a défini les vertus principales du souverain; il voit, dans l'inégalité, la cause la plus importante de tous les désaccords.

Ac ab inaequali manant pleraequae, proinde quod res evertit dissidiumque parit.

Nil, nisi inaequaele est, fit, cum proportio nulla in moderando alios et tribuendo subest.

Personis pariter cum non aequalibus aequa dantur, inaequales sportulaque aequa manet.

Cum neque praefectus rebus par esse regendis, nec moderatori dissona forma potest.

(v. 483-490)

Selon Rakovský, c'est la classe moyenne des citoyens qui constitue le centre d'une bonne société:

Optimus est, qui nec locuples, nec pauper habetur, solus enim prodest civibus ille suis.

$$
\text { (v. 141-142) }
$$

Il proclame les vertus de cette classe et la nécessité d'une érudition, subordonnée au sentiment chrétien. En même temps, selon Rakovský, c'est par cette voie que l'on atteint le bonheur personnel; aux marchands, il attribue le nom de parasites; les serfs pour lui n'ont pas de droits; et les ouvriers donnent leur travail à bail: c'est ainsi qu'il résout la question sociale. En outre, il étudie les raisons des changements et des troubles des États. Dans le poème De magistratu politico, il se reporte à la loi naturelle de l'homme; étant donné la puissance et l'autorité de l'Empereur et de l'État, il incline vers la monarchie.

Juraj Koppai de Driemoty, membre du cercle des lettrés près de Trenčin, publie quant à lui Vita aulica (1580), ${ }^{41}$ la plus violente des critiques sur la classe dirigeante et sur la vie de la cour. Il dédie ce poème à l'archevêque de Prague, Antonius Brus. Il vaut mieux rester exilé, pense-t-il, 
qu'être esclave d'un travail acquis d'une manière véreuse; la richesse nous entraîne dans des mauvais chemins loin des moeurs cultivées:

\section{Exiliumque sequi, sequi et immutabile fatum est melius parto male quam servire labori, est neque virtutis miseris nocuisse colonis. Omnia vincit opum fastus laudisque cupido in vetitum consurgit iter moresque probatos et nisi sacra auri mundique potentia regnat, tanta avidos vexat lucri vesana cupido.}

$$
\text { (v. 38-44) }
$$

Selon lui, la noblesse n'a pas du tout d'entraves; elle fait ce qu'elle veut, ne respecte ni les lois, ni les mandats de Dieu:

Aspiceres, quid enim vanae non improbus ardor aulae audet? Suadetque rapax ad cuncta Tyrannis, nec metuit leges, summi praecepta Parentis caeca videt dirumque nefas transcendere suadet.

(v. 200-203)

Toutefois, ni ces oeuvres, ni d'autres projets n'ont pu entraîner de véritables changements dans la société. De leur côté, les couches sociales les plus basses tentaient bien d'y parvenir au moyen de diverses rébellions: à titre d'illustration, on notera l'insurrection des paysans de Dóža, réprimée en $1514,{ }^{42}$ dont l'impact a été évident en Slovaquie, puis la révolte des mineurs au centre de la Slovaquie en 1525-1526. Mais ces manifestations et les rébellions contre la maison des Habsbourg ne provoquent pas les transformations sociales que souhaitaient leurs chefs.

Les tentatives de réforme avaient été évidentes au cours des époques antérieures, mais à l'aube des temps modernes, elles se sont multipliées de manière inusitée. Comme tout le reste, le christianisme, traversant des écueils différents, a fait l'objet d'une réévaluation. Dans les pays transalpins, en effet, c'est dans le domaine de la religion que la lutte contre l'ordre féodal s'est effectuée. A l'issue de cette lutte, les problèmes sociaux auraient dû se résoudre, puisque la religion et l'Église formaient les principaux appuis où se fondait l'idéologie médiévale. ${ }^{43}$ Dans le domaine religieux, on distingue deux tendances, dont la première, radicale, a touché l'ensemble de l'Europe. C'est d'abord la manifestation publique de Martin Luther en 1517, puis son refus de voir l'Église comme intermédiaire entre Dieu et l'homme. La Réforme cherchait alors un appui dans le christianisme ancien et dans la 
Bible; elle s'appuyait sur les autorités patristiques; et elle s'intéressait à l'érudition et aux sciences naturelles.

C'est Melanchthon qui joue ici un rôle fort important. Dans ses Loci communes theologici de 1521 (réédités en 1535), il cherche à associer la nouvelle Église à la doctrine ancienne et à lui donner une justification et une légitimité théoriques. Ses réformes pédagogiques, inspirées par Johann Sturmius et Pierre de la Ramée, ont trouvé un large écho, non seulement en Bohême, mais aussi en Slovaquie. Un grand nombre d'étudiants provenant de la Slovaquie avaient accès à l'enseignement de Melanchthon à Wittemberg ${ }^{44}$ et, plus tard, ont maintenu un contact littéraire et personnel avec lui.

En outre, en Slovaquie, la Réforme avait beaucoup en commun avec l'humanisme. Il y a donc des traces du mouvement réformé au commencement du seizième siècle chez des humanistes comme Valentin Eck de Lindau, recteur au collège de Bardejov et Leonard Stöckel, recteur à Bardejov, puis à Kežmarok. En 1540, ce natif de Bardejov a écrit le règlement et le programme des études de cette école; il s'agit de l'un des plus anciens documents de ce type en Slovaquie. De même, Stöckel est l'auteur de la Confessio Pentapolitana de 1549.45 Cette confession proposait l'unité religieuse de cinq villes de l'Est de la Slovaquie (Bardejov, Košice, Levoča, Prešov et Sabinov). Quant à la Confessio Heptapolitana, faisant l'unité de sept villes au centre de la Slovaquie, elle révélait jusqu'à un certain point la formation de plusieurs influences du protestantisme en Slovaquie, confirmée par le synode de Žilina en 1610.46 A l'Est de la Slovaquie, un milieu très favorable se développait depuis longtemps grâce aux contacts étroits avec Cracovie et aux relations avec Érasme. Ce dernier connaissait personnellement plusieurs humanistes slovaques, et certains étaient considérés comme ses proches amis. Johannes Antoninus de Košice était d'ailleurs son médecin à Bâle et membre de son cercle d'amis à Cracovie. ${ }^{47}$ Parmi les correspondants d'Érasme, on peut citer entre autres Mikuláš Oláh, ${ }^{48}$ Anton Vrančič, Ján Henkel et Stanislav Turzo. Le caractère de cette correspondance était à la fois privé et public; elle offrait souvent un important moyen social et esthétique d'annoncer ou de propager les idées nouvelles et d'influencer l'opinion publique.

Les rapports entre Érasme et les humanistes slovaques ne sont pas encore assez étudiés. Comme base d'un réexamen, il faudrait consulter l'oeuvre d'Érasme dans les bibliothèques privées et les institutions en Slovaquie. Les catalogues de livres du seizième siècle, publiés jusqu'à maintenant, peuvent servir de point de départ. ${ }^{49}$ On y constate des répétitions: Stultitiae laus - 
Moriae encomium, Adagiorum chiliades, Colloquiorum familiarum opus, Enchiridion christiani militis et De duplici copia verborum. Mais le concile de Trente a mis les écrits d'Érasme à l'Index, les considérant l'oeuvre d'un réformateur, bien que celui-ci n'ait jamais accepté Luther et qu'il ait refusé par exemple de porter un jugement sur le programme des Frères Tchèques (dont l'activité est ressentie aussi en Slovaquie). En traduisant les Saintes Écritures et en faisant la critique et la révision des autorités médiévales et des interprètes des textes sacrés, il a rapproché la théologie des disciplines laïques en soulignant l'importance de la philologie, de la philosophie et de la rhétorique. Les idées d'Érasme étaient très répandues à travers tout le territoire slovaque et elles ont constitué un substrat facilitant l'acceptation de la Réforme. Parallèlement, Érasme met en évidence la notion d' "humanisme chrétien": il s'efforce de modifier l'Église, mais d'une manière modérée, ce qui trouve à se refléter non seulement dans la vie ecclésiastique, mais aussi dans l'enseignement et la culture.

Un des principaux traits caractérisant l'influence novatrice de la Renaissance est l'adoption des langues nationales dans la littérature. La traduction de la Bible devient la norme pour l'ensemble des langues. En Bohême, par exemple, on publie entre 1579 et 1594 à Kralice dans l'imprimerie de l'Unité de Frères une traduction parfaite de la Bible en six tomes. Un groupe de six spécialistes, sous la direction du professeur Ján Blaho, a préparé cette traduction. En plus, le Slovaque Vavrinec Benedikt de Nedožery, professeur à l'Université de Prague, écrit dès 1603 la première grammaire de la langue tchèque. ${ }^{50}$ Dans sa préface, il reproche à ses compatriotes - c'est-à-dire les Slovaques - d'avoir été négligents dans le perfectionnement de leur langue et de faire usage, quand ils parlent le slovaque, d'un mélange de latin et de langue vernaculaire. La Bible en langue tchèque a commencé à se propager relativement tôt parmi les protestants slovaques; ces formes anciennes du tchèque ont été en usage dans la liturgie protestante slovaque jusque dans les années quatre-vingt du vingtième siècle. Elles ont certainement influencé l'évolution de la langue slovaque. C'est donc surtout la littérature slovaque d'allégeance protestante qui tire son origine de cette langue. Toutefois, on ne manquera pas de noter les rapports étroits avec l'Université de Prague qui était au Moyen Age bien fréquentée par les étudiants slovaques. Mais la langue slovaque apparaît beaucoup plus tôt, notamment dans les sermons des prédicateurs slaves (plebanus, concinator sclavonicus ou sclavus) qui, dès le quatorzième siècle, étaient actifs auprès des prêtres allemands et hongrois dans toutes les villes importantes. ${ }^{51}$ Les homélies médiévales ont 
eu d'ailleurs un impact important; ce genre occupe à l'époque des incunables la deuxième place quant à sa fréquence. De nombreuses recherches philologiques et historiques à partir des protocoles et des registres municipaux, des lettres et des chansons documentent l'existence de cette langue ancienne. ${ }^{52}$ L'arrivée de la Renaissance et de la Réforme n'a fait que confirmer cette tendance.

La langue est, selon la conception moderne, l'un des principaux attributs d'une nation. C'est à la Renaissance que s'élaborent les entités nationales en Europe et qu'en Slovaquie un sentiment d'appartenance nationale commence à apparaître. Ce sentiment se développera surtout à l'époque du slavisme baroque à la fin du dix-septième et au commencement du dix-huitième siècle. Dans la suite des formes des communautés humaines (la famille, la tribu, l'ethnie, la nation), la forme de la nation est l'aboutissement. Son évolution marque le passage de la langue familiale à la langue de la nation. ${ }^{53}$ Cette problématique, dans le cas de la Slovaquie, a été rarement traitée. Dans son discours De iustitia et iure,${ }^{54}$ Daniel Basilius distingue la notion de "ius gentium," c'est-à-dire de droit international ou de droit des gens, droit des habitants d'une nation et droit des étrangers. Celle-ci forme le droit privé, "ius privatum," en se juxtaposant à deux autres composantes: le droit civil ("ius civile") et le droit naturel ("ius naturale"). Les nations emploient ce concept de droit privé pour différencier leur territoire et leur empire, pour assurer leur souveraineté et respecter leurs contrats. En Hongrie, la notion de peuple et de nation a coïncidé à cette époque avec celle de nation politique que Štefan Verböci définissait dans un ouvrage intitulé Tripartitum $^{55}$ comme une nation hongroise politiquement commune, une "natio hungarica."

D'après Daniel Rapant, ${ }^{56}$ la nation comme communauté organiquement naturelle et spirituelle, destinée à unir les habitants d'un territoire commun ayant une langue et une culture communes, a commencé à apparaître dans les traités des humanistes lettrés. Bien que les attaches de ces humanistes aient été cosmopolites, leur sentiment national était déjà considérable dès cette époque. Cette notion d'appartenance à une nation commune ressemblait plutôt à un patriotisme local, augmenté d'une dimension confessionnelle. Ainsi, certains lettrés slovaques ont adopté des titres de noblesse comme Peter de Réva, Daniel Basilius de Deutschenberg, ou des surnoms, pour la plupart de forme latine, indiquant la ville ou le pays de leur origine, comme par exemple Pavol Rubigall Pannonius (en Slovaquie le surnom "Pannonius" était employé le plus souvent à la fin du quinzième et au commencement du 
seizième siècle), Ján Antoninus Cassoviensis (de Košice), Peter Fradelius Schemnicensis (de Banská Štiavnica), Ján Sambucus Tyrnaviensis (de Trnava) ou Pannonius, Vavrinec Benedikt Nudozierinus (de Nedožery), Valerianus Mader Trencziniensis (de Trenčin). Et même le médecin Juraj Purkircher Posoniensis ou Pisoniensis a tiré son nom de Presbourg Posonium ou Pisonium: passionné de botanique, il dérivait le nom de cette ville du mot latin pisum (pois).

Par ailleurs, il est intéressant de relire les vers de Ján Sambucus. Dans son Emblemata, ${ }^{57}$ certains vers sont dédiés à sa patrie et à sa ville natale de Trnava ("Tirnaviae patriae meae arma"):

Urbs est Pannonicis fama inclyta, libera regnis,

Tyrnaviam vulgus nomine reque vocat ...

Haec me produxit lucemque videre serenam vitalesque haustus dulcis alumna dedit.

En outre, tout comme par ailleurs Peter Fradelius, Valerián Mader a célébré l'amour de la patrie en écrivant des vers sur le blason de la ville de Trenčin. ${ }^{58}$

On trouve une autre forme de l'élan patriotique dans le poème de Pavol Rubigall, intitulé "Querella Pannoniae ad Germaniam," dans lequel le poète semble porter le sort du pays tout entier. ${ }^{59}$ Il y réclame le secours des Allemands dans la lutte contre les Turcs, suite à la misère qui avait suivi la catastrophe de Moháč et la mort du roi Louis II:

Nam meus ut cecidit saevis Ludovicus in armis

pro patria pugnans proque salute mea,

perpetuo iacui sic tempore semper ab illo

a Mahometigenis undique cincta Getis,

(v. 19-24)

Rubigall exhorte ses compatriotes dans leur lutte contre les Musulmans; ils se sauveront eux-mêmes d'un grand danger tout en gagnant la gloire éternelle:

Ista tuam mentem subeant, Germania, tandem,

sique sapis, dirum quam cito perde genus.

Sic nos in sedes non solum, chara, repones, sed coeli condes nomen in astra tuum.

(v. 279-282)

Dans un autre poème à caractère civil et politique, intitulé "Epistola Pannoniae ad Germaniam," il fait des reproches à l'Allemagne de ne pas être venue en aide à la Hongrie. ${ }^{60}$ De la même manière, Jakub Jakobeus a composé le poème "Gentes Slavonicae lacrumae, suspiria et vota," dans lequel s'affirme un sens 
national aigu. Ce poème est un supplément à une oeuvre aujourd'hui disparue: Viva gentis Slavonicae delineatio (Levoča 1642-1662) ${ }^{61}$ :

Slavorum montes, Slavorum flumina flete!
Flete meos natos, casus magnosque dolores!
Sic volo, sic cupio, Slavorum saucia mater!

(v. 131-133)

La Mère des Slaves personnifiée (Jakobeus prend les Slaves pour des Slovaques en ce qui concerne l'ethnie) se plaint de la situation pitoyable du pays et de la destinée amère de la nation. Simultanément, il commémore le passé glorieux des Slaves et la tradition de la Grande Moravie pour les défendre contre les attaques des autres nations limitrophes, surtout des Allemands, et plus tard des Hongrois. Des thèses similaires apparaissent ailleurs chez Ján Filický. Dans son poème "Ad sphettium,"62 il évoque le souvenir de la gloire passée des Slovaques qui luttaient jadis héroïquement et il constate que depuis ce temps rien n'a changé:

... invenies Slavos virtute et Marte potentes et nihil a proavis degenerare suis.

(v. 13-14)

On distingue aussi un autre type de patriotisme, le patriotisme hongrois de Peter de Réva dans De sacra corona regni Hungariae (Augsbourg, 1613). En outre, un sentiment d'appartenance slave est présent à l'Université de Prague avant la bataille de Biela Hora (1620), notamment chez un certain nombre de professeurs d'origine slovaque: Ján Jesenský, Vavrinec Benedikt, Daniel Basilius ou encore Peter Fradelius. Ceux-ci prennent le parti des Tchèques contre les Allemands, ce qui se traduit par des comportements différents, des rencontres hostiles et même des attaques personnelles. ${ }^{64} \mathrm{Par}$ exemple, Fradelius cherche l'appui de son ami allemand Georg Remus en faisant appel à leur affinité nationale et confessionnelle. ${ }^{65}$ Un pareil sentiment apparaît dans une lettre écrite le 5 février 1575 par le médecin de Presbourg Juraj Purkircher et adressée à son ami Joachim Camerarius junior à Nürnberg. Il lui annonce qu'il vient de se marier et que sa femme, issue d'une ancienne famille allemande, est née à Presbourg. Puis il rappelle ses études universitaires à Padoue et le groupe d'étudiants allemands auquel il s'était joint avec d'autres de ses proches. ${ }^{66}$ L'intérêt pour l'histoire nationale et la célébration des personnages et événements importants qui marquent cette histoire sont ici tout à fait évidents. Ján Sambucus, toujours professeur de médecine et conseiller personnel de l'empereur, est même nommé historiographe aulique de Vienne en 1565.67 
Le progrès culturel n'est ni continu ni uniforme; son évolution diffère selon les régions. Ces différences sont dues à l'absence d'un centre ayant le caractère d'une cour royale ou d'un milieu universitaire (à l'exception de l'activité de courte durée de l'Université Istropolitana de Presbourg au quinzième siècle). Cependant, dans beaucoup de régions de la Slovaquie, il y a des ferments de progrès, cultivés et favorisés par l'aristocratie, les riches bourgeois ou les associations municipales.

A la différence de l'époque médiévale, ce "novum" se produit à plusieurs niveaux. Peu à peu, les éléments du proto-humanisme se présentent sous forme traditionnelle; ils sont incorporés à l'augustinianisme, la Devotio moderna, puis la conscience nationale, du nominalisme au wiklefisme, et enfin le réalisme platonicien. Le retentissement social de ces mouvements est important en ce qui a trait aux rapports avec l'Église et à la résolution des contradictions dans la société. C'est Ján Hus qui, dans la préface de ses sermons, avait très tôt formulé des propositions annonçant des revendications sociales et réformatrices. 68

L'humanisme et ses variantes - l'humanisme de Charlemagne, celui d'Othon $1^{\text {er }}$, les humanismes scolastique, chrétien, utraquiste - sont conçus comme des courants littéraires philologiques orientés sur l'antiquité. Toutefois, il faut se rendre compte que les références fréquentes aux auteurs antiques sont normales à cette époque et qu'un lettré, éduqué par l'université médiévale, s'en préoccupait certainement beaucoup plus qu'un humaniste. Ce dernier en tirait des conclusions différentes. Les formes empruntées à l'antiquité contenaient souvent des idées conservatrices et retardatrices, même si, en fin de compte, elles auraient dû orienter vers une vie active, créatrice et équilibrée, comme le proclamait Érasme. Ce modus vivendi, transmis par la personnalité de Melanchthon, a été mis en valeur dans certaines régions de la Slovaquie. Du point de vue de la forme, du style, de la métrique et du contenu littéraire, il ne s'agit plus simplement d'adopter de grands buts esthétiques. La poésie occupe une place dominante, mais, en dehors des courts genres poétiques habituels, elle tend à solliciter de nouvelles formes d'expression sociales et surtout subjectives, reflétant des sentiments contemporains. Ces transformations sont évidentes dans la poésie slovaque, notamment dans la littérature spirituelle. Outre les poètes éminents comme Martin Rakovský, Ján Sambucus, Juraj Koppai, Ján Filický et Ján Bocatius, qui écrivaient en latin, il faut mentionner, par exemple, Ján Silván et les débuts de l'hymne slovaque, ou Vavrinec Benedikt, ${ }^{69}$ auteur d'une poétique aujourd'hui disparue et fondateur de la prosodie tchèque. Ján 
Silván est considéré comme "le poète des passions humaines et de la souffrance" 70 ; dans ses vers, il évoque souvent sa jeunesse de débauche et sa vie souvent peu vertueuse:

Z hlubokosti Wolám Bože $\mathrm{k}$ tobě

a spomocy twé čekám wždy od tebe

vslyss Bože muog ač gest $\mathrm{z}$ daleka

neb ta propast w nijž wězým gestit'hluboká.

$\left(6^{\mathrm{e}} \text { psaume, } 1^{\mathrm{re}} \text { strophe }\right)^{71}$

D'autre part, grâce en partie aux lettrés étrangers, attirés par les richesses naturelles et l'exploitation minière, le niveau de la vie scientifique augmente en Slovaquie, et un bon nombre d'humanistes commencent à traiter de sciences naturelles. Parmi ces lettrés, on compte Ján Dernschwam, facteur d'Anton Fugger à Banská Bystrica, qui a donné à Juraj Agricola des renseignements sur les mines, la métallurgie et la sidérurgie slovaques. Dans son ouvrage De ortu et causis subterraneorum libri V (Bâle, 1546), il mentionne la région de Spiš et les villes de Smolnik, Banská Bystrica et de Banská Štiavnica. De son côté, intéressé par l'exploitation minière, le célèbre médecin et alchimiste Paracelse a effectué deux voyages en Slovaquie. Juraj Verner, d'origine slovaque, a publié un admirable traité sur les eaux minérales en Hongrie ancienne. ${ }^{72}$ Dans le domaine de l'astronomie, Juraj Joachim Rheticus (mort en 1574), élève de Nicolas Copernic, qui a sauvegardé l'oeuvre de ce dernier, est demeuré quelque temps à Košice. Professeur à l'Université de Prague, Daniel Basilius (1585-1628) s'est lui aussi occupé de la doctrine héliocentrique, et ses thèses sur les problèmes de physique et d'astronomie ont été préservées sous formes de disputes publiées. Basilius a rédigé, entre autres, des almanachs et des annuaires astronomiques, des "ephemerides," et surtout huit grands calendriers. Le plus ancien de ces annuaires date de $1615,{ }^{73}$ tandis que le plus ancien calendrier remonte à $1618^{74}$; ils représentent selon les recherches actuelles en Slovaquie les premiers almanachs en langue nationale vernaculaire. ${ }^{75}$

Par ailleurs, la médecine suivait la conception traditionnelle de Galien. Mais, en 1600, Ján Jessenius a pratiqué la première autopsie à Prague. ${ }^{76}$ L'intérêt pour le règne animal et le règne végétal augmentait; Peter Fradelius $^{77}$ et Juraj Purkircher se sont intéressés à la botanique. Même certains poètes trouvaient une inspiration dans l'observation de la nature: Ján Filický a écrit des vers sur les fourmis et Martin Rakovský sur les écrevisses à pattes rouges. La prose spécialisée commence également à se développer en Slovaquie: les essais, les traités scientifiques, les thèses et les disputationes 
universitaires augmentent en proportion de l'intérêt pour les sciences et de l'évolution des nouvelles disciplines scientifiques. Selon leur contenu et leur importance variée, on peut les répartir en plusieurs genres littéraires où s'entremêlent plusieurs tendances: l'accord entre le sujet et la forme, le choix de la langue, le style et les figures et la composition, de même que les tournures poétiques.

Hors du domaine religieux, l'influence de la Renaissance et de l'humanisme en Slovaquie se remarque avant tout dans l'épanouissement de la culture. On constate donc au seizième siècle une évolution de l'imprimerie, des méthodes d'enseignement et de la laïcisation et un accroissement de la production religieuse en slovaque ou en tchèque slovaquisé. En même temps, la pénétration de la littérature étrangère empreinte de modernité est importante à cause des rapports étroits avec la Bohême, l'Allemagne et l'Italie, et à cause de son impact sur la production locale des livres. Mais, sans une analyse approfondie des manuscrits et des imprimés, dont le prix d'achat décroît graduellement, il est très difficile de mesurer les influences singulières et leur réception en territoire slovaque. D'autre part, les méthodes d'enseignement se perfectionnent au seizième siècle; elles resteront en vigueur jusqu'au dix-huitième siècle. Le réseau des écoles secondaires urbaines ou paroissiales augmente en Slovaquie. Pourtant, beaucoup de lettrés, une fois leurs études terminées, s'installent à l'étranger. L'Église catholique perd sa position dominante (aussi bien dans le domaine économique), l'importance des villes s'accroît, ce qui entraîne une certaine démocratisation de la société slovaque. La vie spirituelle et culturelle, bien que moins abondante, peut déjà se comparer à celle des pays voisins.

Il est impossible de traiter de toutes les questions esthétiques et artistiques, ni de l'ensemble des formes et de leurs contenus. Dans cet article, nous avons voulu mettre en évidence l'impact d'une pensée nouvelle qui aspirait à une transformation de la société et de l'Église et qui a conduit à une prise de conscience des individus et des conditions d'existence de la communauté nationale. Plusieurs principes acceptés durant cette période ont positivement influencé les générations slovaques au cours des siècles ultérieurs. Les postulats de l'harmonie psychique et physique, de l'humanité, du désir de savoir et de connaître, de vivre à l'amiable et en accord avec les principes du christianisme sont néanmoins toujours actuels.

Académie des Sciences de la Slovaquie, Bratislava 


\section{Notes}

1. Le territoire indiqué comme "Uhorsko" ne correspond pas au territoire de la Hongrie d'aujourd'hui. Il s'agit de la Hongrie ancienne qui était peuplée par sept nations différentes qui devaient être égales en droit, d'après l'exhortation du premier roi hongrois Étienne $1^{\text {er }}$ en 1000. STEPHANUS I rex: Kniha mravných ponaučeni svätého Štefana král'a král'ovičovi Imrichovi. Libellus sancti Stephani regis de institutione morum ad Emericum ducem, in Richard Marsina, Legendy stredovekého Slovenska (Banská Bystrica, 1997), pp. 21-32.

2. On peut faire remarquer la fondation de la Société de cuivre de Turzo (Ján de Betlanovce) et de Fugger (Jacob d'Augsbourg) en 1494 à Banská Bystrica, dont les activités sont considérées importantes pour l'évolution du capital européen. Selon le témoignage du chevalier allemand Almud von Harffa, qui a visité l'Égypte et les pays voisins en 1496-1499, la Hongrie a exporté à cette époque-là des métaux précieux d'une valeur de 300000 ducats par an. Cela représentait 100-110 kg. d'or pur. Voir Jozef Vozár, "Rudné baníctvo na Slovensku od 12. storočia do prvého použitia strelného prachu roku 1627," Rudy, 32 (1984), 5, p. 69.

3. Pendant 150 ans, la Slovaquie a été le lieu d'invasions continuelles des Turcs, et ses régions du sud ont formé les quatre "sandžak" qui représentaient l'administration de l'Empire osmane sur le territoire slovaque. Voir Dejiny Slovenska, vol. II (1526-1848) (Bratislava, 1987), pp. 31-35.

4. Posonium (en latin), Prešporok (en slovaque - Bratislava est un nom plus récent), Preßburg (en allemand), Pozsony (en hongrois).

5. Durant la période 1563-1830, dix rois de la famille des Habsbourg et huit épouses royales ont été couronnés au Dôme de Saint-Martin à Presbourg. Voir Štefan Holčik, Korunovačné slávnosti, Bratislava 1563-1830 (Bratislava, 1986).

6. Trnava (ville située à $50 \mathrm{~km}$. de Presbourg) est devenue entre 1635 et 1777 le siège d'une importante université de la Compagnie de Jésus. Après la révocation de cet ordre en 1773 par le décret "Dominus ac redemptor noster" du pape Clément XIV, on a déplacé cette université avec son imprimerie et un tiers des volumes de sa bibliothèque à Buda (aujourd'hui Budapest en Hongrie).

7. Strigonium (= Ostrihom, Esztergom) se trouve en Hongrie; une partie de la Slovaquie de l'Est était administrée par l'archevêque de Jáger (Eger, en Hongrie).

8. Par exemple, Presbourg, Trnava, Banská Bystrica, Banská Štiavnica, Kremnica, Levoča, Bardejov, Sabinov. Les Allemands y ont convoqué pour la première fois le roi Belo IV après les invasions pernicieuses des Tartares en 1241-1242 et en 1244. Dans la région de Spiš, on a commencé à constituer les conseils administratifs indépendants des Allemands saxons. La deuxième colonisation, dite des Allemands saxons, s'est déroulée au seizième siècle. Ces colons ont reçu des privilèges avantageux au détriment de la population autochtone; ceux-ci ont été souvent la cause de troubles. Voir Dejiny Slovenska, vol. I (do roku 1526) (Bratislava, 1986), p. 223.

9. Ibid., p. 427. 
10. Mária Kohútová, Demografický a sídlištny obraz západného Slovenska (Bratislava: Historické štúdie, 1990), p. 73.

11. L'armée de Louis II Jagellon, roi de la Hongrie et de la Bohême, a affronté celle du sultan Sélim (Sulejman, Soliman) I ${ }^{\mathrm{er}}$ près de Moháč (Mohácz en Hongrie), le 29 août 1526. La mort de Louis II et la victoire des Turcs a signifié en vérité la fin du royaume hongrois médiéval; dans la politique européenne, la question turque a été souvent soulevée avec insistance.

12. Ferdinand $\mathrm{I}^{\mathrm{er}}$ Habsbourg, frère de la Reine Marie, veuve de Louis II Jagellon, prétendant au trône hongrois, a été élu d'après le droit naturel Roi de Hongrie, le 17 décembre 1526, au Parlement de Presbourg; et le 3 novembre 1527, comme Ján Zápol'ský l'année précédente, il était couronné à Stoličny Belehrad (Székesfehérvár). Cette loi de succession se reportait au traité signé par l'empereur Maximilien $I^{\text {er }}$ de Habsbourg avec les rois de la Hongrie et de la Bohême Ladislaus II Jagellon et son fils Louis II à Vienne en 1515.

13. Le Voïvode transilvain Ján Zápol'ský (mort en 1540) a été élu d'après le droit usuel roi de Hongrie au parlement convoqué à Stoličny Belehrad (Székesfehérvár en Hongrie), le 10 novembre 1526 par le parti national ou pro-hongrois. Le sultan Sélim le soutenait aussi. Voir Dejiny Slovenska, vol. II (1526-1848), p. 22.

14. Il faut mentionner les insurrections des grands magnats et des Voïvodes transilvains: Štefan Bočkai (mort en 1606), Gabriel Betlen (mort en 1629), Juraj l ${ }^{\text {er }}$ Rákoci (mort en 1648). Voir Ibid., pp. 42-165.

15. Jozef Minárik, Renesančná a humanistiká literatúra. Svetová, česká, slovenská (Bratislava, 1985), p. 7.

16. Jozef Minárik, Ibid.; Jozef Kuzmík, Slovník autorov slovenských a so slovenskými vzt'ahmi za humanizmu, I-II, 1976.

17. Dejiny Slovenska, vol. I (do roku 1526), pp. 308-312.

18. On peut citer, par exemple, Johannes Vitéz, plus tard archevêque d'Esztergom et précepteur de Mathias Corvin; le précepteur de Johannes Vitéz était un humaniste italien du nom de Pier Paolo Vergerio Vegesius qui vivait en Hongrie.

19. "Studium generale ad instar studii Bononiensis"; voir Karol Rebro, "Istropolitana a Bologna," in Humanizmus a renesancia na Slovensku v 15.-16. storoči (Bratislava, 1967), pp. 15-20.

20. Branislav Varsik, Národnostný problém Trnaskej univerzity (Bratislava, 1938),p. 6; Antonio Bonfini, Historia Pannonica sive Hungaricarum rerum decades, IV (Kolin, 1690), dec. IV, lib. I, p. 391.

21. Imrich Kotvan, Inkunábuly so slovenskými vzt'ahmi (Bratislava, 1974), pp. 47-52.

22. Albert de Berzeviczy, Béatrice d'Aragon, reine de Hongrie (1457-1508), vols. I-II (Paris, 1911), pp. 105-238. Son attitude active devant son mari avec qui elle était une partenaire égale, sa participation aux débats savants à la cour royale sont présentés dans l'ouvrage d'Antonio Bonfini, conservé grâce à Ján Sambucus, Symposion sive Trimeron sive De virginitate et puditia coniugali dialogi III (Francfort, 1621). 
23. D'après le contrat de mariage, les villes minières en Slovaquie centrale appartenaient à la reine de Hongrie.

24. Tibor Kardos, "Devotio Moderna na Academii Istropolitane," in Humanizmus a renesancia na Slovensku, pp. 29-38.

25. Eva Frimmová, "Der Humanismus in Pressburg am Ausgang des Mittelalters," in Verdrängter Humanismus - Verzogerte Aufklärung, vol. I, 1 (Philosophie in Osterreich [1400-1650]) (Klausen-Leopoldsdorf), 272-292.

26. 2105 étudiants de la Slovaquie se sont inscrits à l'Université de Cracovie entre 1400 et 1525. Voir Samuel Osuský, "Naši študenti na Krakovskej univerzite od 1400-1525," Viera a veda, 2 (1931), pp. 174-178; Samuel Osusky, "Naši bakalári, magistri a dekani na Krakovskej univerzite od 1400-1525," Viera a veda, 2 (1931), pp. 226-233.

27. Jusqu'à la seconde moitié de 1450, on a immatriculé 19780 étudiants, dont 4151 Hongrois, Tchèques et Polonais qui se déclaraient de la Natio Hungarica; 2929 d'entre eux étaient certainement hongrois. Voir Károly Schrauf, Magyarországi tanulók a bécsi egyetemen (Budapest, 1892).

28. L'Université Charles IV à Prague était l'université la plus fréquentée en Europe centrale à la fin du quatorzième et au début du quinzième siècle, surtout par les Hongrois. Après les guerres des Hussites, cet intérêt a diminué, mais à la fin du quinzième et au début du seizième siècle, les étudiants slovaques (on les identifie dans les matricules en terme de la nation "Sclavus" ou "Slavus") recherchaient toujours cette université, notamment à cause des affinités culturelles et linguistiques. Voir Branislav Varsik, Slováci na pražskej univerzite do konca stredoveku (Bratislava, 1926), s. 37; Husiti a reformácia na Slovensku do žilinskej synody (Bratislava, 1932), pp. 138-147.

29. Július Sopko, Stredoveké latinské kódexy v slovenských knižniciach (Martin, 1981); Július Sopko, Kódexy a neúplne zachované rukopisy $v$ slovenských knižniciach (Martin, 1986); Július Sopko, Stredoveké latinské kódexy slovenskej provenience v Mad'arsku a v Rumunsku (Martin, 1982); Jean-Marie Olivier et Marie-Aude Monégier du Sorbier, Catalogue des manuscrits grecs de Tchécoslovaquie (Paris, 1983); Imrich Kotvan, Inkunábuly na Slovensku (Martin, 1979); Imrich Kotvan et Eva Frimmová, Inkunabuly zo slovenských knižnic v zahraničných inštitúciách(Martin, 1996).

30. Peter Kuklica, "Ciceros Beziehung zur Literatur," in Graecolatina et orientalia. Zborník Filozofickej fakulty Univerzity Komenského, 9-10 (1977-1978), p. 85; Daniel Škoviera, "Virgile en Slovaquie à l'époque de l'humanisme latin," Listy filologické, 106 (1983), pp. 58-62; Imrich Kotvan, "Prírodovedné tlače 15. storočia v slovenských knižniciach," in Humanizmus a renesancia na Slovensku v 15.-16.storici, pp. 436-444.

31. Július Sopko, "La littérature antique dans les manuscrits slovaques médiévaux," in Graecolatina et orientalia. Zborník Filozofickej fakulty Univerzity Komenského, 5 (1973), p. 167.

32. Miloslav Okál, "Hieronymus Balbus," Zprávy Jednoty klasických filologu, 13 (1971), 91-117.

33. Jozef Minárik, Renesančná a humanistická literatúra, pp. 73-74.

34. Vojtech Bucko, Mikuláš Oláh a jeho doba 1493-1568 (Bratislava, 1940). 
35. Daniel Škoviera, "Das humanistische Lehrbuch De versificationis arte opusculum von Valentinus Ecchius Lindaviensis," Graecolatina et orientalia. Zborník Filozofickej fakulty Univerzity Komenského, 17-18(1985-1986), 45-65; Daniel Škoviera, "Antike Autoren und Autoritäten in dem versologischen Handbuch des Humanisten Valentinus Ecchius Lindaviensis," Graecolatina et orientalia. Zborník Filozofickej fakulty Univerzity Komenského, 19-20 (1987-1988), 31-51.

36. Jan Macek, Italská renesance (Prague, 1965).

37. Jozef Minárik, Renesančna a humanistická literatúra, p. 15.

38. Daniel Škoviera, "Der Dialog De reipublicae administratione von dem Humanisten Valentinus Ecchius," Graecolatina et orientalia. Zborník Filozofickej fakulty Univerzity Komenského, 23-24 (1992), 73-84.

39. Miloslav Okál, Život a dielo Martina Rakovského, vols. I-II (Martin, 1979, 1988).

40. Philipp Melanchthon, In Aristotelis aliquot libros Politicos commentaria (Paris, 1536).

41. Miloslav Okál, Juraj Koppay: Zobrané spisy (Bratislava, 1980); Georgius Koppay. Vita aulica (Prague, 1580), 295 hexamètres, in Okál, pp. 158-204.

42. Hobereau de la Transilvanie, Juraj Dóža a été nommé commandant de la croisade contre l'empire osmane. Cette croisade a été déclarée par l'archevêque d'Esztergom et le cardinal Thomas Bakóc au nom du pape Léo X: elle s'est transformée en insurrection des paysans. Voir Dejiny Slovenska, vol. I (do roku 1526), pp. 419-421.

43. Jozef Minárik, Renesančná a humanistická literatúra, pp. 14-15.

44. Un grand intérêt pour les universités protestantes allemandes est documenté: entre 1522 et 1600, environ 1000 étudiants de la Hongrie, dont 360 venaient de la Slovaquie, ont fini leurs études à l'Université de Wittemberg. Voir Dejiny Slovenska II (1526-1848), p. 128.

45. Daniel Škoviera, "Leonard Stöckel und die Antike - Die klassische Bildung eines Schulhumanisten," Graecolatina et orientalia. Zborník Filozofickej fakulty Univerzity Komenského, 23-24 (1992), 73-84.

46. La Confessio Pentapolitana a paru a la suite de la publication de la loi contre les anabaptistes et les sacramentaires en 1548. Voir Branislav Varsik, Husiti a reformácia na Slovensku do Žilinskej synody, pp. 172-185. En même temps, il faut noter que c'est en 1608 au parlement de Presbourg, au moment de l'élection de Mathias de Habsbourg, roi de Hongrie, qu'on a accepté la loi permettant l'émancipation en droit des peuples dans les villes libres et garantissant la liberté de confession pour tous les habitants. Voir Lexikón slovenských dejín (Bratislava, 1997), p. 68.

47. Daniel Škoviera, "Johannes Antoninus Cassoviensis (1495-1547) - ein aus der Slowakei gebürtiger Freund von Erasmus von Rotterdam," Graecolatina et orientalia. Zborník Filozofickej fakulty Univerzity Komenského, 13-14 (1981-1982), 55-71; "Johannes Antoninus Cassoviensis (1495/9-1547/9) — ein aus der Slowakei gebürtiger Freund von Erasmus von Rotterdam," Graecolatina et orientalia. Zborník Filozofickej fakulty Univerzity Komenského, 15-16 (1983-1984), 57-74. 
48. Vojtech Bucko, Op. cit.; Michal Svatos et Martin Svatoš, Živá tvár̆ Erasma Rotterdamského(Prague, 1985).

49. Helena Saktorová, Klára Komorová, Emilia Petrenková et Ján Agnet, Tlače 16. storočia vo fondoch Slovenskej národnej knižnice Matice slovenskej (Martin, 1993); Helena Saktorová, Klára Komorová et Emilia Petrenková, Tlače 16. storočia v piaristických knižniciach(Martin, 1997); Ján Smetana et Jozef Telgársky, Katalóg tlači 16. storočia v Čaplovičovej knižnici v Dolnom Kubine (Martin, 1981); Vojtech Strelka, Tlače 16. storočia v piaristickejknižnici v Trenčine (Martin, 1996); Mária Vieriková, Tlače 16. storočia v zbierkach Ústrednej knižnice Slovenskej akadémie vied (Bratislava, 1996); Imrich Kotvan, Slaviká XVI. storočia Univerzitnej knižnice v Bratislave (Bratislava,

50. Vavrinec Benedikt Nedozersky, Grammaticae Bohemicae ad leges naturalis methodi conformatae et novis numeris illustratae ac distinctae libri duo (Prague, 1603).

51. Jozef Sasinek, "Slovenski reprezentanti duchovného života Bratislavy v XVI. a XVII. stor.," in Slovanská Bratislava (Bratislava, 1948), pp. 61-157.

52. Ján Dorul'a, Slováci v dejinách jazykových vzt'ahov (Bratislava, 1977); Ladislav Šimoič, "Z knih mesta Skalice zo XVI. a XVII. storocia," Linguistica slovaca, I (1941), 130-153.

53. Pavel Ondrus, "Národ, jazyk a vývin spoločnosti," Philologica. Sborník Filozofickej fakulty Univerzity Komenského, 15 (1963), pp. 3-5.

54. Daniel Basilius, Disputationum Iustinianearum prima De iustitia et iure (Prague, 1612). (Ius civile Bohemicum: Tria architectonica iuris praecepta sunt: honeste vivere, alterum non laedere, suum cuique tribuere).

55. Stephanus Werböczy, Tripartitum opus iuris consuetudinarii inclyti regni Hugariae (Vienne, 1517). Werböczy y a codifié le droit usuel de la noblesse hongroise après la répression de l'insurrection des paysans sous la direction de Juraj Dóža en 1514.

56. Frantisek Bokes, "Počiatky národného prebudenia," in Slovenská vlastiveda, vol. IV, Dejiny (Bratislava, 1946), pp. 130-131.

57. Jån Sambucus, Emblemata (Anvers, 1579).

58. Miloslav Okál et Eva Tkáčiková, Báseñ na znak mesta Trenčina. Valerián Mader (Trencin, 1998).

59. Paulus Rubigallus, Querella Pannoniae ad Germaniam (Wittemberg, 1537), in Miloslav Okál, Paulus Rubigallus. Carmina (Leipzig, 1980), pp. 1-11.

60. Paulus Rubigallus, Epistola Pannoniae ad Germaniam recens scripta (Wittemberg, 1545), in Miloslav Okál, Op. cit., pp. 41-53; Pavol Rubigall, Nový list Panónie Germánii, in Miloslav Okál, Pavol Rubigall. Opis cesty do Konštantinopolu a iné básne (Bratislava, 1985), pp. 47-64.

61. Jozef Minárik et Mária Vyvíjalová, Jakub Jakobeus (ca 1591-1645) (Bratislava, 1963).

62. Ján Filický, Carminum libri duo (Bâle, 1614).

63. Le 8 novembre 1620 , la dynastie des Habsbourg a définitivement vaincu les États tchèques en Bohême et la persécution des protestants y a commencé. 
64. Eva Frimmová, Daniel Basilius (1585-1628). Život a dielo (Bratislava, 1997).

65. Heinrich Kunstmann, Die Nürnberger Universität Altdorf und Böhmen (Cologne-Graz, 1963), pp. 112-115.

66. Miloslav Okál, Georgius Purkircher. Operae quae supersunt omnia (Budapest, 1988), p. 163.

67. Anton Vantuch, Ján Sambucus. Život a dielo renesančného učenca (Bratislava, 1975).

68. Frantisek Šmahel, "Husitstvi - humanismus - renesance," in Humanistická konference (Prague, 1966), pp. 46-47.

69. Oto Kolárik, Vavrinec Benedikt Nedozerský (Martin, 1965), p. 33.

70. Ján Mišianik, Jozef Minárik, Milena Michalcová et Andrej Melicharčik, Dejiny slovenskej literatúry (Bratislava, 1958), p. 118.

71. Dieu, je t'appelle de la profondeur / et j'attends toujours l'aide de Toi / mon Dieu, exauce-moi, si lointain / parce que l'abîme, où je suis enfoui, est bien profond. (Ces vers sont écrits en ancien tchèque slovaquisé.)

72. Juraj Wernher, De admirandis Hungariae aquis hypomnemation (Bâle, 1549); Hypomnemation de aquis in Scepusio admirandis (Vienne, 1551).

73. Daniel Basilius, Minucý nowá a pranostika s pilnosti sepsaná ... k léta Páně, 1615 (Prague, 1614?).

74. Daniel Basilius, Nowý a starý s pranostikou a historiemi kalendár̆ hospodářský a kancelárský ku potřebĕ ouredniküm, pisarùm, prokuratorùm, kupcum a obchody wšelijaké wedoucim. K létu Pánè 1618 (Prague, 1617?).

75. Voir supra n. 64.

76. Josef Polišenský, Jan Jesenský-Jessenius (Prague, 1965).

77. Peter Fradelius, In laudem botanices (Nuremberg, 1612). 